\title{
THE S-PROCESS IN THE YELLOW SYMBIOTIC AG DRACONIS
}

\author{
KATIA CUNHA \\ Observatório Nacional, Rio de Janeiro, Brazil \\ VERNE V. SMITH \\ University of Texas at El Paso, TX 79968, U.S.A. \\ and \\ McDonald Observatory \\ University of Texas at Austin, TX 78712, U.S.A. \\ AND

\section{ALAIN JORISSEN} \\ Institut d'Astronomie, Université Libre de Bruxelles, Belgium
}

\begin{abstract}
An abundance analysis of the yellow symbiotic system AG Draconis reveals it to be a metal-poor $\mathrm{K}$ giant $([\mathrm{Fe} / \mathrm{H}]=-1.3)$ which is enriched in the heavy $s$-process elements. This star thus provides a link between the symbiotic stars and the binary barium and $\mathrm{CH}$ stars which are also $s$-process enriched. These binary systems, which exhibit overabundances of the heavy elements, owe their abundance peculiarities to mass transfer from thermally-pulsing asymptotic giant branch stars, which have since evolved to become white-dwarf companions of the cool stars we now view as the chemically peculiar primaries. A comparison of the heavy-element abundance distribution in AG Dra with theoretical nucleosynthesis calculations shows that the $s$-process is defined by a relatively large neutron exposure $\left(\tau=1.3 \mathrm{mb}^{-1}\right)$, while an analysis of the rubidium abundance suggests that the $s$-process occurred at a neutron density of about $2 \times 10^{8}$ $\mathrm{cm}^{-3}$. The derived spectroscopic orbit of AG Dra is similar to the orbits of barium and $\mathrm{CH}$ stars. Because the luminosity function of low-metallicity $\mathrm{K}$ giants is skewed towards higher luminosities by about 2 magnitudes relative to solar-metallicity giants, it is argued that the lower metallicity $\mathrm{K}$ giants have larger mass-loss rates. It is this larger mass-loss rate that drives the symbiotic phenomena in AG Dra and we suggest that the other yellow symbiotic stars are probably low-metallicity objects as well.
\end{abstract}

No text received 


\section{Discussion}

North: What is the orbital period of this system?

Cunha: A CORAVEL orbit yields $P=549 \pm 7 \mathrm{~d}$, in agreement with the value obtained by Mikołajewska et al. $(1995, A J, 109,1289)$. 